\title{
Decreasing Boarders in the Emergency Department By Reducing Clerical Work in the Discharge Process of In-Hospital Patients - An Interrupted Time-Series Analysis
}

\section{Diego Marques Moroço}

Universidade de São Paulo Hospital das Clínicas da Faculdade de Medicina de Ribeirão Preto:

Universidade de Sao Paulo Hospital das Clinicas da Faculdade de Medicina de Ribeirao Preto

Antonio Pazin-Filho ( $\sim$ apazin@fmrp.usp.br)

Universidade de São Paulo Faculdade de Medicina de Ribeirão Preto: Universidade de Sao Paulo Faculdade de Medicina de Ribeirao Preto https://orcid.org/0000-0001-5242-329X

\section{Research article}

Keywords: Crowding, Emergency Department, Patient discharge, Length of stay, Six sigma and lean, hospital quality, Outpatient Clinics, Hospital, Electronic Health Records, Digital Signature, Business Process Management, Interrupted time series

Posted Date: June 9th, 2021

DOI: https://doi.org/10.21203/rs.3.rs-587650/v1

License: (c) (i) This work is licensed under a Creative Commons Attribution 4.0 International License. Read Full License 


\section{Abstract}

Background: Emergency Department (ED) boarding is related to in-hospital patients' discharge. New techniques implemented in hospital institutions, such as digital signature, offer an improvement on these processes.

Goals: Evaluate the impact of expediting patient's discharge after medical orders with the number of patients diverted to ED.

Methods: We conducted a quasi-experimental study before and after an intervention. It consisted of an encrypted digital signature used to reduce clerical work and expedite the patient's release from the institution after medical discharge. We used Interrupted Time Series Analysis based on administrative data (number of hospital discharge, bed turnover, the time between medical discharge and the appointment's closing) from 2011 to 2019 using.

Results: The interrupted time series analysis for the time from medical order to virtually hospital discharge showed an immediate change in level (Coefficient $\beta 2$-3.6 hours - 95\% confidence interval -3.9; -3.4 - Lag1), but not a difference in the slope of the behavior of the post-intervention curve ( $\beta 30.0005$ coefficient - $95 \%$ confidence interval $-0.0040 ; 0.0050$ - Lag1). For the number of diverged patients to ED, we observed no immediate change in level (Coefficient $\beta 2-0.84$ patients - $95 \%$ confidence interval -0.33 ; 0.16 - Lag1), but a difference in the slope of the behavior of the post-intervention curve ( $\beta 30.0005$ coefficient - 95\% confidence interval $-0.0040 ; 0.0050$ - Lag1).

Conclusion: Reducing clerical work and so expediting patient discharge was associated with a decrease of potential boarders to ED.

\section{Background}

Emergency Department (ED) boarding is the output of several processes[1]. One of these processes is Hospital Out Clinic ( $\mathrm{HOC}$ ) patients considered for unscheduled hospital admission during their visits. If the patient is not having beds available when the hospital admission, the patient is diverged to ED for waiting and having treatment started[2, 3].

Our institution is a Brazilian tertiary referral public teaching hospital with coverage of 4,5 million inhabitants in the northeast region of Sao Paulo. We have around 3,000 external visits (HOC) every weekday, and diversion of unscheduled hospital admission to ED is a common problem[4].

Our hospital implemented an in-house developed electronic health record (EHR) which is continuously actualized and grants us the opportunity to customize processes. We use business process management (BPM) tools to implement any change in the EHR and succeed in other areas of study as sepse[5]. We recently reviewed the discharge process using a through output method. We realized that the physician's 
role in the first steps of the process included many variabilities, considering the diversity of teams and our institution's teaching characteristics.

Otherwise, the patient discharge after medical order was the final step and took seven hours on average, mainly due to more standardized clerical work but required by Brazilian regulations. We hypothesized that reducing this time would be possible by using nurse encrypted digital signature (DS) as a trigger in the EHR to allow safe discharge and interruption of several procedures, such as sending meals and medications to the floor after the patient has left the hospital. Changing this step would also imply more beds available sooner, avoiding diversion to ED.

\section{Methods}

We conducted a quasi-experimental study, before and after an intervention, to expedite the patient's release from the institution after medical discharge. We performed the analysis based on administrative data (number of hospital discharges, bed turnover, the time between medical discharge and the closing of the appointment) from January 1, 2011, up to December 31, 2019. We used Interrupted Time-Series Analysis (ITS) to evaluate the discharge process intervention after medical order in the diversion of unscheduled hospital admissions to the ED. We used data provided by the Clinical Hospital of the Ribeirão Preto Medical School of the University of São Paulo - Brazil - and our institution's Ethical Committee approved the study (CAE 96424318.2.0000.5440).

We designed the BPM current process before January 1, 2017 (before) as detailed in Figure 1(A) and the change after that (Figure $1(B)$ ) using Bizage ${ }^{\circledR}$. The intervention consisted of simplifying the clerical work. We used a nurse-encrypted digital signature to trigger canceling prescriptions and nutrition to be sent to the floor and inform the administrative control that the patient was leaving. The nurse also documented any medications discharged with the patient. The encrypted digital signature introduced in the EHR on January 1, 2017, guaranteed safe discharge.

We excluded data after December 312019 due to COVID-19 pandemic changes in our institution[6]. We hypothesized that the intervention would be immediate and sustained in time in our models. Since the duration of in-hospital stay is associated with different outcomes, we first performed the ITS analysis for the whole group and stratified it into two groups ( $\leq 7$ or $>7$ days).

We also recovered the average number of daily patients diverged to ED by month during the same study time and treated them equally with ITS to evaluate the intervention's impact.

We used itsa command routine in Stata $15 \AA$ to conduct ITS analysis and to construct the graphics. The itsa command routine estimates the parameters according to the following equation + _t. Yt is the aggregated outcome variable measured at each equally spaced time point $t, T$ is the time since the start of the study, Xt is an indicator variable representing the intervention, and XtTt is an interaction term. In a single-group study, $\beta 0$ represents the intercept or starting level of the outcome variable. $\beta 1$ is the slope or trajectory of the outcome variable until the introduction of the intervention. $\beta 2$ represents the change in 
the level of the outcome that occurs in the period immediately following the intervention's introduction. $\beta 3$ represents the difference between preintervention and postintervention slopes of the outcome. Thus we look for significant $p$-values in $\beta 2$ to indicate an immediate treatment effect and in $\beta 3$ to demonstrate a treatment effect over time. Comparing groups follows the same rules but adding interaction terms[7].

\section{Results}

The interrupted time series analysis for the time from medical order to virtually hospital discharge showed an immediate change in level (Coefficient $\beta 2$-3.6 hours - 95\% confidence interval -3.9; -3.4 Lag1), but not a difference in the slope of the behavior of the post-intervention curve ( $\beta 30.0005$ coefficient - $95 \%$ confidence interval $-0.0040 ; 0.0050$ - Lag1). When we stratified by in-hospital stay, there was no change in the results (Figure $2 \mathrm{~A}$ ).

For the number of diverged patients to ED, we observed no immediate change in level (Coefficient $\beta 2-0.84$ patients - $95 \%$ confidence interval $-0.33 ; 0.16$ - Lag1), but a difference in the slope of the behavior of the post-intervention curve ( $\beta 30.0005$ coefficient - $95 \%$ confidence interval $-0.0040 ; 0.0050$ - Lag1) - Figure $2 \mathrm{~B}$.

According to the years studied, the number of patients discharged by the day's hour is demonstrated in histograms (Figure 3). Before the intervention, there was a bimodal distribution, with a considerable amount of discharges occurring after $19 \mathrm{~h} 00$ (2011-2016), the time to nurse shift change in our institution. After the intervention, we can see a normal distribution shifted to the left, with most discharges occurring between $15 \mathrm{~h} 00$ and 16h00 (2017-2019).

\section{Discussion}

We showed that a simple intervention based on encrypted digital signature as a trigger of events in an EHR could reduce the time to discharge after medical order around a median of 7 hours by reducing clerical work. Furthermore, this change was immediate and sustained after the intervention. Finally, a slight and persistent decrease in the number of patients diverged for ED waiting for an available bed on the floor.

Reducing boarders in the ED is a complex problem. The phenomenon is associated with several causes that assume different intensities depending on the institution studied. Therefore, identifying the leading causes to solve is paramount. In our institution, unscheduled hospital admission indicated to HOC patients was a workable BPM evaluation problem. The main problem was guaranteeing safety discharge after medical order according to Brazilian legislation regarding clerical work[8]. Before 2017, Brazilian law obliged healthcare personal to ensure that everything was correct before the patient left the hospital. After 2017, Brazilian regulations started to accept the encrypted digital signature as a valid alternative. We implemented this solution to all healthcare personnel and used it as the primary intervention in this project. 
This intervention had an immediate and sustained effect, reducing 7 hours of the hospital bed turnover. In 2013, The Advisory Board Company (www.advisory.com) estimated a reduction of 0.25 days in the duration of hospital stay for an institution of 600 beds would provide an extra 25 beds available in 24 hours. This amount of beds is what we should expect to accommodate the unscheduled hospital admissions of HOC patients.

Even though the reduction in time to discharge was immediate, the same did not occur to the number of patients diverted to the ED. There could be several explanations for this finding. One of those is an institutional culture (a "reflex") to diverge patients to ED instead of looking for an available bed on the floor. In our institution, each specialty has some designated beds for their use. The "new" bed that the intervention provided was not always one specialty with a patient needing in-hospital admission. We corrected that by implementing an allocation beds central dashboard and guaranteed the teams that "their" beds would be available again in 24 hours if they "lend" it to the specialty in need[9]. Another reason is that some patients must require intensive care unit, positive or negative isolation beds (for cases of tuberculosis or immune depressed conditions, respectively), which are more scarce in the institution.

Besides the impact of expediting the patient discharge, shifting to the left of the leaving hour is significant. Our patients come from small cities around and depend on working hour transportation for going home after discharge. If the discharge occurs after $17 \mathrm{~h} 00$, there is no transportation, the patient has to remain in the hospital up to the following day, and we could not use the bed for another patient waiting in the ED. This problem is aggravated during weekends when this transportation is not available. We attributed the bimodal distribution before the intervention to the nurse duty shifty. The shift to the left and assuming a normal distribution after the intervention corroborates this. Other authors showed similar findings $[10,11]$.

Our approach to avoiding boarder by expediting discharge after medical order is a pull forward strategy since the available bed will "pull" waiting for patients to the floor. Another process recently reviewed in the literature is a push-forward one, represented by the transfer of patients to a lounge on the floor, so the floor staff will try to guarantee an available bed[12]. This push-forward strategy still lacks definitive evidence of effectiveness. Our pull-forward approach seems more logical since it addresses the floor staff's needs instead of putting pressure on them. We acknowledged that our strategy steel requires further studies for a definitive cause-effect relationship. Still, we should consider that differences among institutions, including their culture, should be considered while searching for solutions to avoid boarders in ED.

Our findings are following others. Mustafa et al. demonstrated that the patients diverged to ED from HOC who indicate an unscheduled admission are an essential contributor to crowding. Furthermore, patients with delayed discharge occupied up to $15 \%$ of the hospital beds[2]. Muhammad et al. presented similar results, emphasizing the role that elderly patients represent in this situation[13]. Silva et al. presented similar data in Brazil and pointed to the role that teaching hospitals such as ours could have[14]. 
Ideally, following patients' flow in a thorough output model, including more active measures such as a dedicated person, would bring more immediate results in reducing boarders[15]. Nevertheless, our institution has limited resources, and the solution implemented is very cost-effective. After the present study's findings, as previously mentioned, we implemented a dedicated central to follow up patient flow, including a hospitalist that can intervene proactively to reduce LOS[16, 17]. Newer strategies such as discrete events simulation could work in tandem with BPM to evaluate an intervention's impact before studying it in the field[18].

\section{Abbreviations}

BPM - Business Process Management

DS - Digital Signature

ED - Emergency Department

HER - Electronic Health Record

HOC - Hospital Out Clinic

ITS - Interrupted Time-Series Analysis

LOS - Length of Stay

\section{Declarations}

Ethics approval and consent to participate - Ribeirao Preto Medical School University Hospital University of Sao Paulo - Ethical Committee approved the study (CAE 96424318.2.0000.5440).

Consent to publish - Since we are using retrospective administrative data, we obtained waive of consent from the Ethical Committee

Availability of data and materials - We extracted data from our institutional Electronica Health Record; Data are restricted and can be obtained from the authors by request which had to be approved by the Ethical Committee.

Competing interests - authors declare that there are no competing interests.

Funding - There was no funding besides the consent of the institutional for providing the data. The results of software remodeling were incorporate with no charges to the institutional EHR.

Authors' Contributions - Both authors contributed to extracting and analyzing the data and compounding the manuscript.

Acknowledgements - none

Author's Information - 
Diego Marques Moroço - Master in Healthcare System Management - Ribeirão Preto Medical School University of São Paulo

Antonio Pazin-Filho - Full Professor of Emergency Medicine, Internal Medicine Department, Ribeirão Preto Medical School - University of São Paulo - apazin@fmrp.usp.br (Corresponding Author)

\section{References}

1. Morley C, Unwin M, Peterson GM, Stankovich J, Kinsman L. Emergency department crowding: A systematic review of causes, consequences and solutions. PLOS ONE. 2018;13:e0203316.

2. Mustafa F, Gilligan P, Obu D, O'Kelly P, O'Hea E, Lloyd C, et al. 'Delayed discharges and boarders': a 2year study of the relationship between patients experiencing delayed discharges from an acute hospital and boarding of admitted patients in a crowded ED. Emerg Med J. 2016;33:636-40.

3. Powell ES, Khare RK, Venkatesh AK, Van Roo BD, Adams JG, Reinhardt G. The Relationship between Inpatient Discharge Timing and Emergency Department Boarding. J Emerg Med. 2012;42:186-96.

4. Adolfi Júnior MS, Pallini FM, Pessotti $H$, Wolf CM, Patelli HT, Capeli RD, et al. Emergency medical coordination using a web platform: a pilot study. Rev Saúde Pública. 2010;44:1063-71.

5. Pires HHG, Neves FF, Pazin-Filho A. Triage and flow management in sepsis. Int J Emerg Med. 2019;12:36.

6. Moreira HT, Volpe GJ, Rezek UC, Mendonça PC de, Teixeira GC de A, Santos BM dos, et al. Telemedicina em Cardiologia para Seguimento Ambulatorial de Pacientes com Alto Risco Cardiovascular em Reposta à Pandemia de COVID-19. Arq Bras Cardiol. 2021;116:153-7.

7. Linden A. Conducting Interrupted Time-series Analysis for Single- and Multiple-group Comparisons. Stata J Promot Commun Stat Stata. 2015;15:480-500.

8. Li X, Niu J-W, Ma J, Wang W-D, Liu C-L. Cryptanalysis and improvement of a biometrics-based remote user authentication scheme using smart cards. J Netw Comput Appl. 2011;34:73-9.

9. Martinez DA, Kane EM, Jalalpour M, Scheulen J, Rupani H, Toteja R, et al. An Electronic Dashboard to Monitor Patient Flow at the Johns Hopkins Hospital: Communication of Key Performance Indicators Using the Donabedian Model. J Med Syst. 2018;42:133.

10. Khanna S, Sier D, Boyle J, Zeitz K. Discharge timeliness and its impact on hospital crowding and emergency department flow performance: Discharge Timeliness and Its Impact. Emerg Med Australas. 2016;28:164-70.

11. Kravet SJ, Levine RB, Rubin HR, Wright SM. Discharging Patients Earlier in the Day: A Concept Worth Evaluating. Health Care Manag. 2007;26:142-6.

12. Franklin BJ, Vakili S, Huckman RS, Hosein S, Falk N, Cheng K, et al. The Inpatient Discharge Lounge as a Potential Mechanism to Mitigate Emergency Department Boarding and Crowding. Ann Emerg Med. 2020;75:704-14. 
13. Majeed MU, Williams DT, Pollock R, Amir F, Liam M, Foong KS, et al. Delay in discharge and its impact on unnecessary hospital bed occupancy. BMC Health Serv Res. 2012;12:410.

14. Silva SA da, Valácio RA, Botelho FC, Amaral CFS. Fatores de atraso na alta hospitalar em hospitais de ensino. Rev Saúde Pública. 2014;48:314-21.

15. Lyons J, McCaulley L, Maronian N, Hardacre JM. A targeted initiative to discharge surgical patients earlier in the day is associated with decreased length of stay and improved hospital throughput. Am J Surg. 2019;217:419-22.

16. Messler J, Whitcomb WF. A History of the Hospitalist Movement. Obstet Gynecol Clin North Am. 2015;42:419-32.

17. Ferreira AJ, Laurindo MC, Moraes A de A, Cerantola R, Barbosa LB, Pereira JG da C, et al. A implantação do Núcleo Interno de Regulação em Hospital Público Terciário. Med Ribeirao Preto Online. 2019;52:16-23.

18. Qin S, Thompson C, Bogomolov T, Ward D, Hakendorf P. Hospital occupancy and discharge strategies: a simulation-based study: Hospital occupancy and discharge. Intern Med J. 2017;47:8949.

\section{Figures}




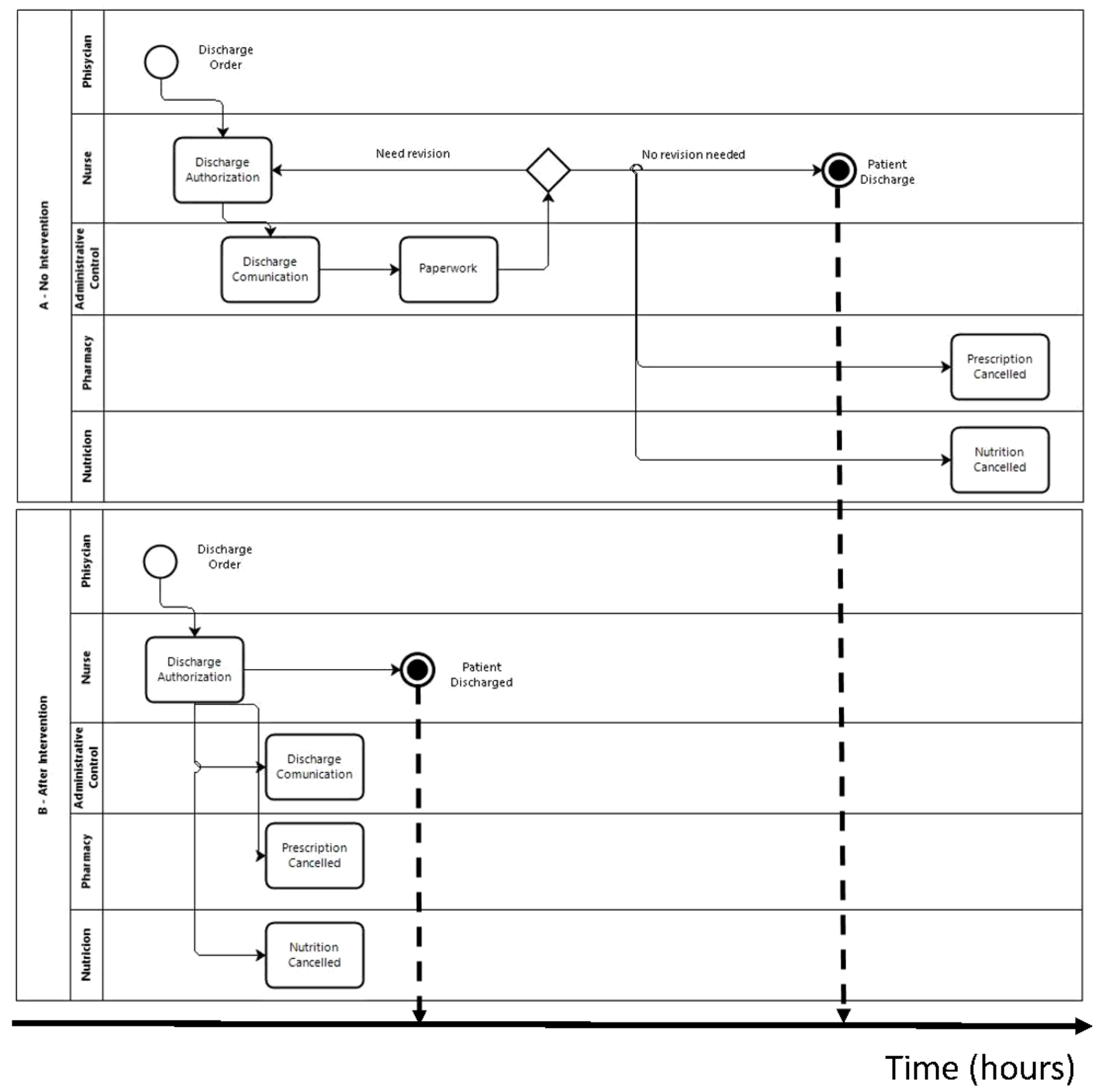

Figure 1

Discharge process after medical order before (A) and after (B) the intervention. 


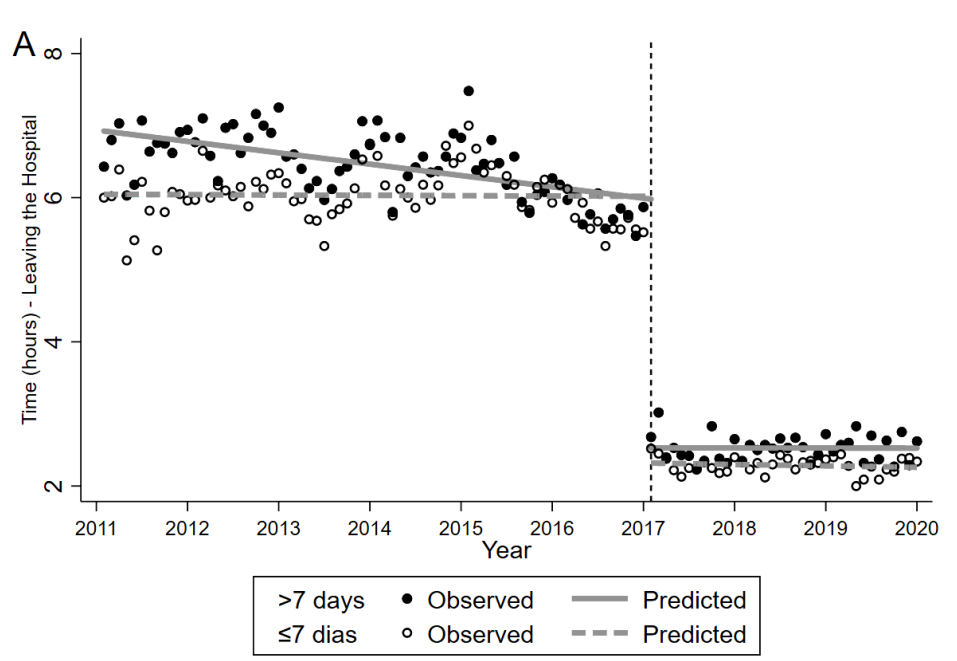

Regression with Newey-West standard errors - lag(1)

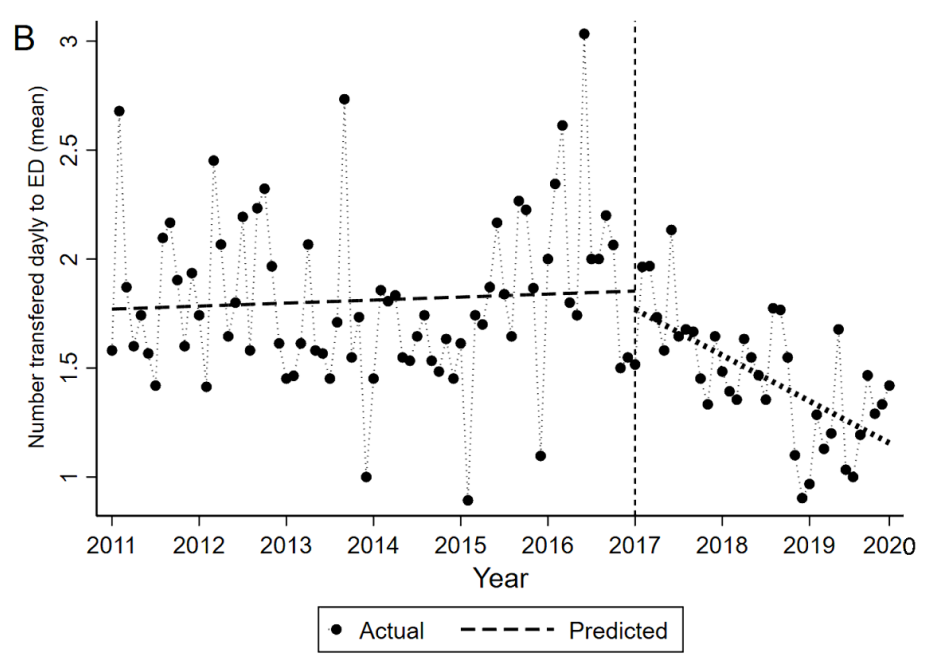

Regression with Newey-West standard errors - lag(1)

\section{Figure 2}

Interrupted Time-Series analysis of time to discharge after medical order $(A)$ and mean number of diverted patients to the Emergency Department (B) before and after the intervention $-p<0.05$ for both scenarios.

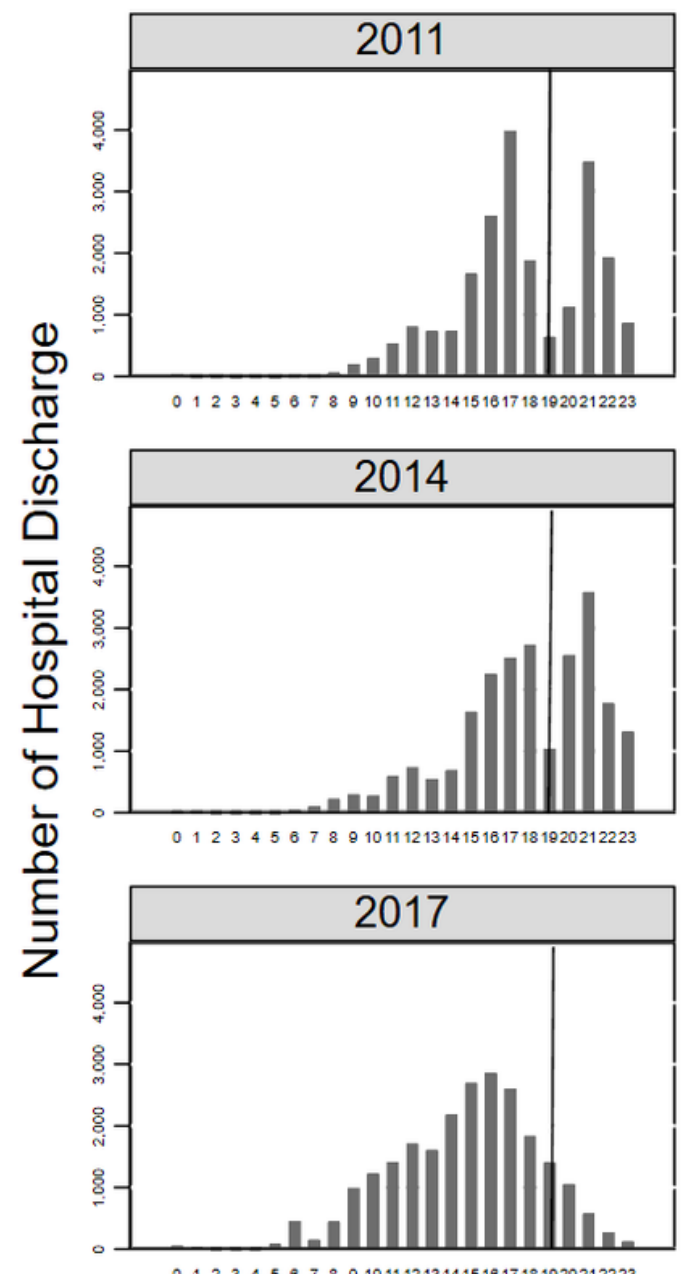

01234567891011121314151617181920212223

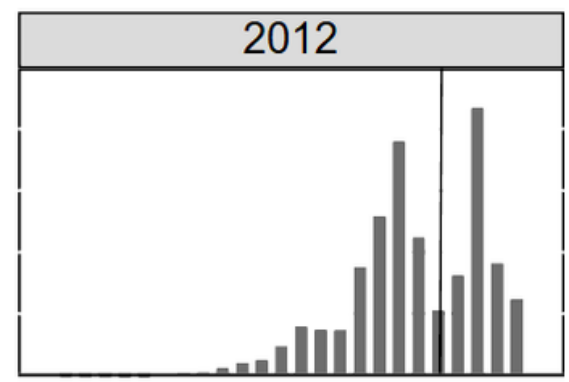

01234567891011121314151617181920212223

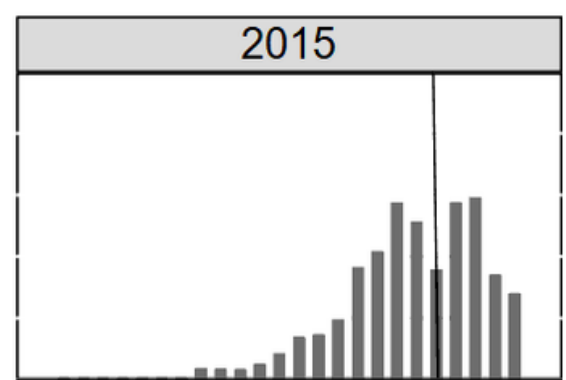

01234567891011121314151617181920212223

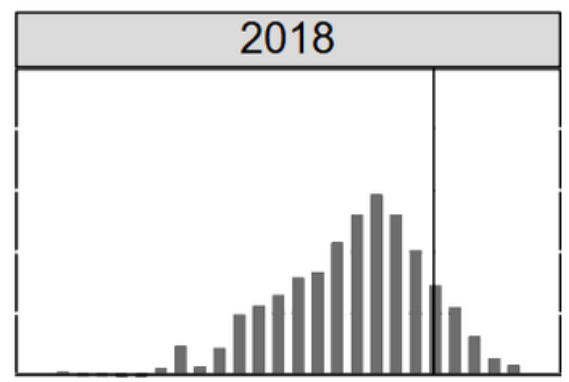

01234567891011121314151617181920212223

Time of day (hours)

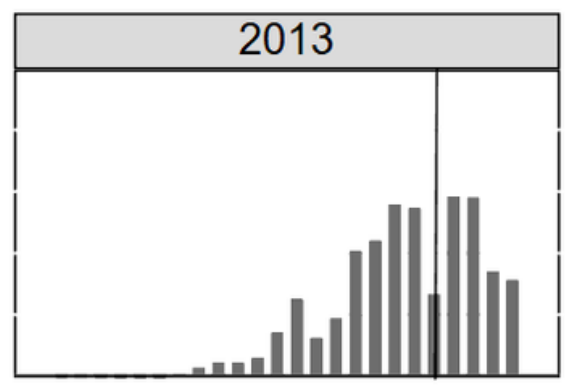

01234567891011121314151817181920212223

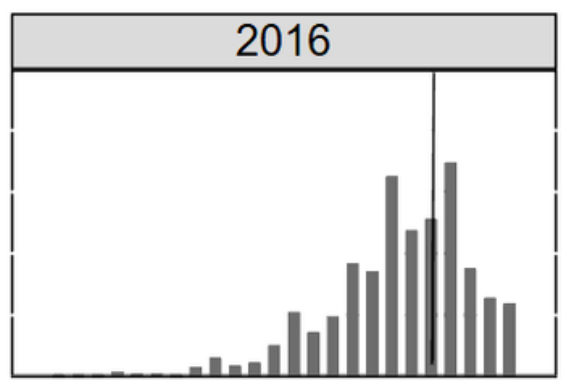

01234567891011121314151617181920212223

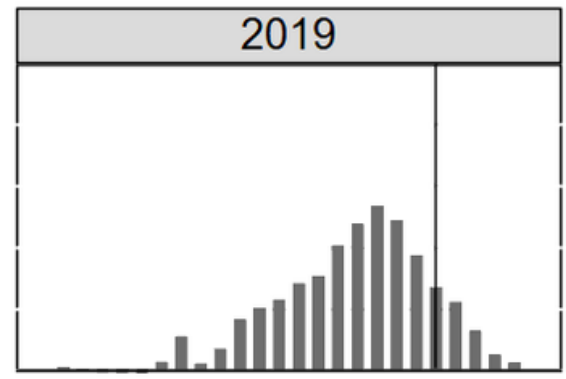

01234567891011121314151617181920212223 
Figure 3

Histograms of Number of patients discharged accordingly to the hour of day by year of study. The line marks the nurse duty shift at $19 \mathrm{~h} 00$. 